\title{
Prevalence of Community Acquired Uropathogens and their Antimicrobial Susceptibility in Patients from the Urology Unit of A Tertiary Care Medical Center
}

\author{
Manzoor Ahmad ${ }^{1}$, Pushkar Kumar ${ }^{2}$ D , Asfia Sultan² (D), Anees Akhtar ${ }^{2}$, \\ Bhanu Chaudhary ${ }^{2}$ and Fatima Khan ${ }^{2 *}$ (D) \\ ${ }^{1}$ Department of Surgery, Jawaharlal Nehru Medical College, Aligarh, Aligarh - 202002 U.P. India. \\ ${ }^{2}$ Department of Microbiology, Jawaharlal Nehru Medical College, Aligarh, Aligarh - 202002 U.P. India.
}

\begin{abstract}
Urinary tract infections (UTI) are one of the most common community acquired infections and can also present with similar lower urinary tract symptoms (LUTS). Moreover, UTI can be a complication of these urological diseases. Thus, this study was conducted in patients with LUTS to find out the prevalence of uro-pathogens and their antibiotic susceptibility pattern so that appropriate antibiotics can be started on clinical suspicion of UTI. The study was conducted over a period of 12 months. Culture and susceptibility of urine specimen was done as per standard microbiological guidelines. Apart from the growth of common bacteria with their antibiogram, Methicillin-resistant Staphylococcus aureus (MRSA), high level aminoglycoside resistance (HLAR) was investigated among Enterococci, and metallo beta-lactamases(MBL) production was production was investigated among gram-negative pathogens. Out of 407 urine samples included in the study, 80 (19.6\%) samples showed bacterial growth. The commonest isolate was $E$. coli 44 (55\%). Majority of the isolates were multidrug resistant with two E.coli strains showing pan-resistance to the first line drugs tested. Carbapenem resistance was seen in $67.2 \%$ of all gram negative isolates tested. Metallo beta-lactamases production was found to be highest among the Klebsiella isolates. Among the Enterobacterales, highest susceptibility was noted to Fosfomycin $(87.7 \%)$ followed by Nitrofurantoin $(62.7 \%)$. Similarly in gram positive group, highest susceptibility was again to Fosfomycin followed by Nitrofurantoin. Overall the prevalence of MDR is increasing however Fosfomycin or Nitrofurantoin, both oral antibiotics, can be the considered for starting empirical antibiotic therapy.
\end{abstract}

Keywords: UTI, antimicrobial resistance, urology, LUTS, BOO

*Correspondence: fatimasalmanshah@gmail.com; +91 7060317021

(Received: June 30, 2020; accepted: August 24, 2020)

Citation: Ahmad M, Kumar P, Sultan A, Akhtar A, Chaudhary B, Khan F. Prevalence of Community Acquired Uropathogens and their Antimicrobial Susceptibility in Patients from the Urology Unit of A Tertiary Care Medical Center. J Pure Appl Microbiol. 2020;14(3):2009-2015. doi: 10.22207/JPAM.14.3.40

(C) The Author(s) 2020. Open Access. This article is distributed under the terms of the Creative Commons Attribution 4.0 International License which permits unrestricted use, sharing, distribution, and reproduction in any medium, provided you give appropriate credit to the original author(s) and the source, provide a link to the Creative Commons license, and indicate if changes were made. 


\section{INTRODUCTION}

Urinary tract infections are considered as one of the most common infections developing in a community setting. Estimates show that more than 150 million episodes of UTIs occur annually world over ${ }^{1}$. They are a major cause of outpatient attendance as well as indoor admissions. It is also responsible for a major share of antibiotic consumption worldwide with negative socio-economic repercussions ${ }^{2,3}$. One of the major concerns for last many years is the fear of developing antibiotic resistance due to non-judicious use by health care professionals ${ }^{4}$. As the urine culture sensitivity report takes more than 2 days, clinicians often face difficulties in deciding the right antibiotic to be prescribed in an outpatient setting.

In Urology Outpatient clinics, we see lot of patients with lower urinary tract symptoms (LUTS) which refers to a group of symptoms like frequency, urgency, hesitancy, dribbling, poor flow of urine and straining at urination ${ }^{5}$. These symptoms start developing in $5^{\text {th }}$ decade of life with higher prevalence in males than females ${ }^{6,7}$. One of the commonest causes of LUTS in males is Benign prostatic hypertrophy (BPH) which produces these symptoms secondary to bladder outlet obstruction ${ }^{8}$. It is estimated that about half of the male population has histopathological evidence of BPH with the prevalence increasing to around $90 \%$ at the start of ninth decade of life $^{9}$. Urinary tract infection is also one of the complications of bladder outlet obstruction due to various causes some of which requires surgical intervention at some point of their clinical course ${ }^{10}$.
Both UTI and bladder outlet obstruction can present with similar LUTS. Thus UTI needs to be ruled out in patients with LUTS before commencing cause specific medications or advising operation. Thus this retrospective study was designed to know the prevalence of uropathogens and their susceptibility pattern in patients presenting with LUTS in our Urology OPD. The ultimate aim is to make a local protocol for starting empirical treatment whenever a patient presents in outpatient clinic with clinical features suggestive of UTI.

\section{MATERIALS AND METHODS}

\section{Patients and study design}

The study was conducted over a period of 12 months (March 2019 to February 2020) in the Urology unit of Department of Surgery and Department of Microbiology, JNMCH, AMU, Aligarh. This study was approved by Institutional ethics committee and informed consent of all patients was obtained.

\section{Specimen Collection and processing}

Urine samples were collected from all new patients of age 15 years and above presenting with lower urinary tract symptoms like pain and burning during urination, frequency, urgency, supra pubic pain etc. Freshly collected mid-stream clean catch urine samples were collected from the non-catheterized, alert, conscious patients with above mentioned indications. The urine samples were processed as soon as possible after being received in the laboratory. The urine samples were plated by semi-quantitative method with standard loop technique on CLED Agar incubated

Table 1. Distribution of microbiological flora in two age groups in urine specimen

\begin{tabular}{|c|c|c|c|c|c|}
\hline \multirow[t]{2}{*}{ Organism } & \multicolumn{2}{|c|}{ Age $15-60$ years } & \multicolumn{2}{|c|}{ Age $>60$ years } & \multirow[t]{2}{*}{ Total $=80$} \\
\hline & Male (\%) & Female (\%) & Male (\%) & Female (\%) & \\
\hline E.coli & $19(43.18)$ & 15 (34.10) & $9(20.45)$ & $1(2.27)$ & $44(55)$ \\
\hline Klebsiella sp. & $11(73.33)$ & $2(13.33)$ & $2(13.33)$ & - & 15 (18.75) \\
\hline Citrobactor sp. & $2(40)$ & $2(40)$ & $1(20)$ & - & $07(8.8)$ \\
\hline Enterococcus sp. & $4(57.14)$ & $2(28.57)$ & - & $1(14.28)$ & $05(6.2)$ \\
\hline CONS & $3(75)$ & $1(25)$ & - & - & $04(5)$ \\
\hline Pseudo sp. & $2(100)$ & - & - & - & $02(2.5)$ \\
\hline S. aureus & $2(100)$ & - & - & - & $02(2.5)$ \\
\hline Proteus vulgaris & $1(100)$ & - & - & - & $01(1.25)$ \\
\hline Total & & & & & $80(100)$ \\
\hline
\end{tabular}


at $37^{\circ} \mathrm{C}$ overnight. The growth of organisms (single or double) based on colony count $\left(\geq 10^{3}\right)$ were taken ${ }^{11}$. Isolates significant clinically, as described in the standard guidelines of ICMR with some modifications, were included in the study ${ }^{12}$.

\section{Exclusion criteria}

Patients with catheters or ureteral stents, recent history of antibiotic consumption, inadequate urine samples ( $<10 \mathrm{ml}$ urine), samples collected from urine bag, specimens collected more than 2 hours before submission, specimens submitted in leaking, or dirty unsterile containers and specimens revealing growth of more than two types of bacteria on culture.

\section{Antibiotic susceptibility testing}

Antimicrobial susceptibility testing of all isolates was performed as per clinical laboratory standards institute guidelines ${ }^{13}$ on Mueller Hinton agar by Kirby-Bauer disk diffusion method.. The antimicrobial discs were procured from Hi-Media Laboratories, Mumbai, India.

\section{Enterobacterales isolates}

Cotrimoxazole (1.25/23.75 $\mu \mathrm{g})$, Amikacin $(30 \mu \mathrm{g})$, Ceftriaxone $(30 \mu \mathrm{g})$, CeftriaxoneSulbactam $(75 \mu \mathrm{g}, 1: 1)$, Cefixime $(5 \mu \mathrm{g})$, AmoxicillinClavulanic acid $(20 \mu \mathrm{g} / 10 \mu \mathrm{g})$, Fosfomycin $(200 \mu \mathrm{g})$, Nitrofurantoin $(300 \mu \mathrm{g})$, Norfloxacin $(10 \mu \mathrm{g})$ and Meropenem (10 $\mu \mathrm{g})$ as the first line drugs. Pathogens resistant to the first line drugs were taken as multi-drug-resistant and were tested against second line drugs, Piperacillin-Tazobactam (100:10 $\mu \mathrm{g})$, Tobramycin $(10 \mu \mathrm{g})$, Imipenem (10 $\mu \mathrm{g})$, Polymyxin B $(300 \mu \mathrm{g})$ and Colistin $(10 \mu \mathrm{g})$.

\section{Pseudomonas spp.}

Piperacillin-Tazobactam (100:10 $\mathrm{g})$, Meropenem $(10 \mu \mathrm{g})$ and Colistin $(10 \mu \mathrm{g})$, Amikacin $(30 \mu \mathrm{g})$, Aztreonam $(30 \mu \mathrm{g})$, Cefepime $(30 \mu \mathrm{g})$, Nitrofurantoin $(300 \mu \mathrm{g})$, Gentamycin $(10 \mu \mathrm{g})$, Levofloxacin $(5 \mu \mathrm{g})$ and Ceftazidime $(30 \mu \mathrm{g})$.

Staphylococcus aureus and CONS isolates

Amikacin (30 $\mu \mathrm{g})$, Amoxicillin $(20 \mu \mathrm{g})$,

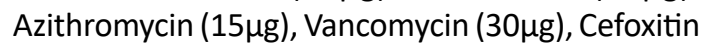
$(30 \mu \mathrm{g})$, Cotrimoxazole $(1.25 / 23.75 \mu \mathrm{g})$, Fosfomycin $(200 \mu \mathrm{g})$, Nitrofurantoin $(300 \mu \mathrm{g})$, Norfloxacin (10 $\mu \mathrm{g})$.

\section{Enterococcus species}

Ampicillin $(10 \mu \mathrm{g})$, Azithromycin (15 $\mu \mathrm{g})$, Doxycycline(30 $\mu \mathrm{g})$, Fosfomycin $(200 \mu \mathrm{g})$, High Content Gentamycin $(120 \mu \mathrm{g})$, High Content Streptomycin $(300 \mu \mathrm{g})$, Vancomycin $(30 \mu \mathrm{g})$, Nitrofurantoin $(300 \mu \mathrm{g})$ and Norfloxacin

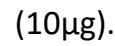

\section{Detection of Metallo beta-lactamases}

Imipenem resistant isolates were tested for Metallo beta-lactamases (MBL) production by modified Hodge test and Double Disc synergy test using EDTA $^{14}$.

Screening for methicillin resistance in Staphylococcus species, high-level aminoglycoside resistance in Enterococci and Vancomycin resistance in Enterococci

Test was performed on Muller Hilton agar with $4 \% \mathrm{NaCl}$ using Cefoxitin (30 $\mu \mathrm{g}$ ) disc. Isolates showing a zone size $<22 \mathrm{~mm}$ for Staphylococcus aureus and $<25 \mathrm{~mm}$ for Coagulase negative staphylococcus species were considered resistant ${ }^{13}$.

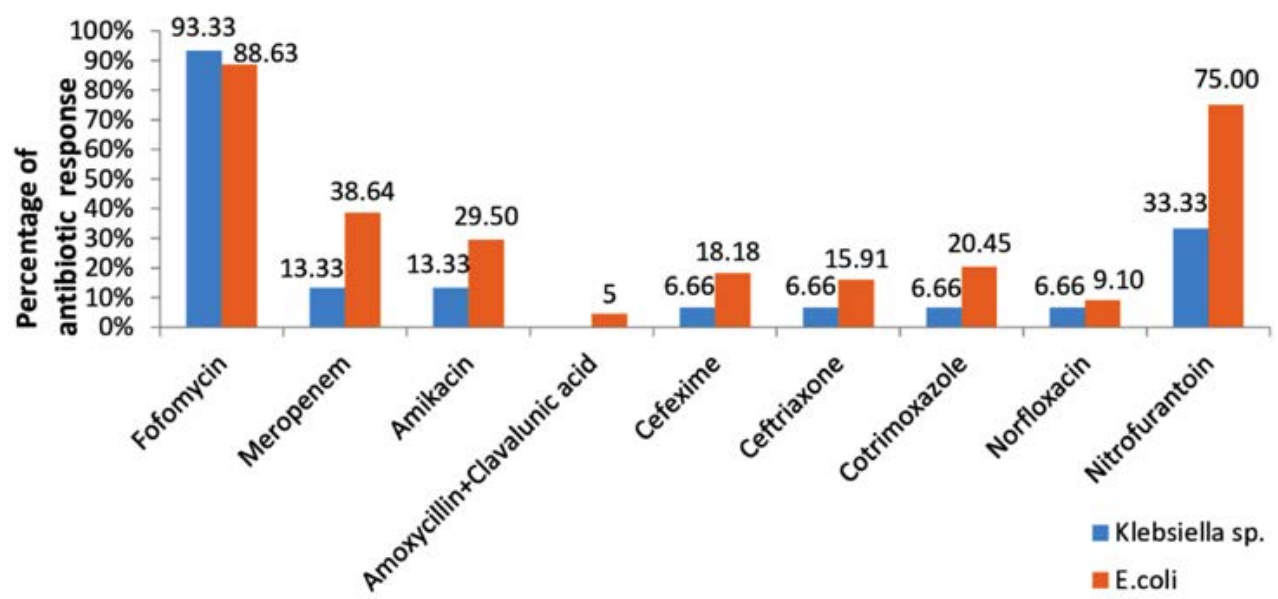

Fig. 1. Antibiotic sensitivity pattern of Klibsiella sp. and E.coli. 
For Enterococcal isolates, high-level aminoglycoside resistance (HLAR) was tested using high content gentamycin $(120 \mu \mathrm{g})$ and high level streptomycin $(300 \mu \mathrm{g})$. Zone size $\geq 10 \mathrm{~mm}$ is considered as sensitive and zone size $<6 \mathrm{~mm}$ (no zone) is considered to be resistant (HLAR) ${ }^{14}$ and for the detection of VRE, $30 \mu \mathrm{g}$ disc of Vancomycin disc is used along with the routine susceptibility testing and zone of inhibition $\leq 14$ are considered as VRE. Those with intermediate zones are confirmed by MIC methods ${ }^{13}$.

\section{RESULTS}

Out of 407 urine samples included in the study, 316 (77.6\%) were from males and 91 (22.4\%) were from female patients. Of these $80(19.6 \%)$ samples showed bacterial growth \& 327 (80.4\%) samples were sterile. Culture positivity was higher in females $26.4 \%$ (24 out of 91 ) as compared to males $17.7 \%$ (56 out of 316 ).

Of all positive urine cultures, 56 (70\%) patients were males while 24 (30\%) were females. Male to female ratio was 2.3:1. Categorization was also done on the basis of age group of patients; Group 1 consisted of the patients with the age of 15-60 years and group 2 with patient's age above 60 years. Majority of the culture positive patients were in group $1 ; 66(82.5 \%)$ consisting of $44(66.7 \%)$ males and $22(33.3 \%)$ females. Out of total 14 elderly patients in Group 2 with UTI, 12 (85.71\%) were males and $2(14.28 \%)$ were females. Out of the eighty culture positive specimen majority 67 (83.8\%) were gram negative isolates while gram positive organisms were cultured in $13(16.2 \%)$ specimen. The frequency of isolation of these organisms is shown in Table 1. Almost all the specimens included the study showed monomicrobial growth. The most common isolate was E coli 44 (55\%) followed by Klebsiella species 15 (18.8\%). Among Gram positive isolates, Enterococci was the commonest 07 (8.8\%). Other organisms, which were identified included Citrobactor species 07 (6.2\%), Coagulase negative Staphylococcus species 04 (5\%), Pseudomonas species 02 (2.5 $\%$ ), Staphylococcus aureus 02 (2.5\%), and Proteus species 01 (1.25\%).

E.coli was the most common bacteria isolated in all age and gender groups. Among the Enterobacterales, highest susceptibility was noted to Fosfomycin (87.7\%) followed by Nitrofurantoin (62.7\%). Almost all the gram negative strains (94.4\%) were resistant to Amoxicillin-Clavulanic acid.

Majority of the isolates were multidrug resistant with two E.coli strains showing resistance to all the first line drugs tested. Of these two isolates tested for $2^{\text {nd }}$ line drugs, one was susceptible only to Colistin whereas the other one

Table 2. Antimicrobial Sensitivity Pattern of Gram Negative Bacterial Isolates in percentages

\begin{tabular}{lcccccccccc}
\hline & Fo & Mrp & Ak & Amc & Cfm & Ctr & Cot & Nx & Nit \\
\hline E.coli $(\mathrm{n}=44)$ & 88.6 & 38.6 & 29.50 & 4.5 & 18.18 & 15.91 & 20.45 & 9.10 & 75 \\
Klebsiella sp. $(\mathrm{n}=15)$ & 93.33 & 13.33 & 33.33 & 0 & 6.66 & 6.66 & 6.66 & 6.66 & 33.33 \\
Citrobactor sp. $(\mathrm{n}=5)$ & 60 & 40 & 20 & 20 & 20 & 40 & 20 & 40 & 60 \\
\hline
\end{tabular}

Fo $=$ Fosfomycin, $\mathrm{Mrp}=$ Meropenem, $\mathrm{Ak}=$ Amikacin, $\mathrm{Amc}=$ Amoxycillin + Clavalunic acid, $\mathrm{Cfm}=$ Cefixime, $\mathrm{Ctr}=$ Ceftriaxone, Cot $=$ Co-trimoxazole, $\mathrm{Nx}=$ Norfloxcin, Nit $=$ Nitrofurantoin

Table 3. Antimicrobial Sensitivity Pattern of Gram Positive Bacterial Isolates in percentages

\begin{tabular}{lccccccccccccc}
\hline Uropathogens & Fo & Ak & Cot & Nx & Nit & HG & HS & Va & Amp & Azm & Amx & Cx & Do \\
\hline $\begin{array}{l}\text { Enterococcus } \\
\text { sp. }(n=7)\end{array}$ & 100 & 0 & 0 & 14.28 & 71.42 & 85.72 & 57.14 & 100 & 57.14 & 14.29 & 0 & 0 & 28.57 \\
CONS $(n=4)$ & 100 & 100 & 50 & 25 & 100 & - & - & 100 & - & 75 & 75 & 50 & - \\
S. aureus $(n=2)$ & 100 & 100 & 50 & 50 & 100 & - & - & 100 & - & 50 & 50 & 50 & - \\
\hline
\end{tabular}

$\mathrm{HG}=$ High sensitive gentamycin, $\mathrm{HS}=$ High sensitive streptomycin, Va = Vancomycin, Amp = Ampicillin, Azm= Azithromycin, $\mathrm{Cx}$ $=$ Ceftriaxone, $\mathrm{Do}=$ Doxycycline, Fo =Fosfomycin, Ak = Amikacin, Cot $=$ Co-trimoxazole, $\mathrm{Nx}=$ Norfloxcin, Nit $=$ Nitrofurantoin, $\mathrm{Cx}=$ Cefoxitine, $\mathrm{Amx}=$ Amoxicillin.

Journal of Pure and Applied Microbiology 
was sensitive to all the $2^{\text {nd }}$ line drugs tested i.e., Colistin, Tigecycline, Polymixin B and Gentamycin. Out of 15 Klebsiella isolates, one tested for $2^{\text {nd }}$ line antimicrobials which showed sensitivity to Tigecycline only and was resistant to Polymixin B, Colistin, Gentamycin and Ceftazidime. The antibiotic resistance profile of E. coli and Klebsiella sp. is depicted in Fig. 1.

The only Proteus vulgaris isolate was found to be sensitive to Fosfomycin and Nitrofurantoin and was resistant to Meropenem, Amikacin, Cefixime, Co- Trimoxazole and Norfloxacin.

Carbapenems resistance was seen in $67.2 \%$ of all gram negative isolates. Metallo-betalactamases production was found to be highest among the Klebsiella isolates followed by E.coli and Citrobactor species. The antibiogram of the different bacterial organisms isolated from the urine specimen is reported for gram negative and gram positive isolates in Table 2 and Table 3 respectively.

Both of the Pseudomonas growth was sensitive to Colistin and one of them was also sensitive to Meropenem, Piperacillin-Tazobactum and Aztreonam. However both of them were resistant to Cefepime, Gentamycin, Levofloxacin, Ceftazidime, and Nitrofurantoin.

Enterococcus was the most common bacteria in the Gram positive group. All the seven were uniformly (100\%) sensitive to Fosfomycin and Vancomycin followed by Nitrofurantoin (84.2\%). Amongst the staphylococcal isolates, all were consistently susceptible to Fosfomycin, Amikacin, Nitrofurantoin and Vancomycin. Half of the isolates of Staphylococcus aureus and coagulase negative species showed susceptibility to Cefoxitin. The remaining $50 \%$ were thus considered to be methicillin resistant. High level aminoglycoside resistance was noted among 6 out of 7 Enterococcal isolates.

Multidrug resistance was noted in $91.2 \%$ amongst all the isolates tested. MDR frequency was higher among the gram negative isolates i.e. $94 \%$ (63 out of 67) as compared to gram positive isolates $76.9 \%$ (10 out of 13). MDR was highest in Klebsiella sp. (100\%) and Enterococcus species (100\%) followed by E.coli (93.2\%) and Citrobactor sp. $(80 \%)$.

\section{DISCUSSION}

This study was done to identify the prevalence of UTI and antibiotic sensitivity pattern in patients presenting with LUTS in the Urology clinic of J. N. Medical College, AMU, Aligarh. In our study, males were affected more than females, which is contrary to various previously published studies $^{1,2,15,16}$. The reason for this can be explained by the fact that three fourth of our study subjects were males. Positive culture was found more commonly in the younger age group that is less than 60 yrs. It may be because the condition of bladder outlet obstruction (BOO) which commonly affects the elderly can also present with similar set of lower urinary tract symptoms. This reason can be supported by the fact that majority of our study population were males in whom $\mathrm{BOO}$ is commoner than in females.

In this study E. coli (55\%) was the most common organism isolated followed by Klebsiella sp. (18.8\%). The commonest gram positive isolate was Enterococcus sp. (8.8\%). In the gram negative group, Fosfomycin and Nitrofurantoin has good coverage which confirms the findings from other studies $^{1,2}$. There was poor coverage by Amikacin as compared to previous study from the same center ${ }^{2}$. This may be due to higher empiric use of Amikacin in the region in the indoor patients because of higher sensitivity in previous years leading to the development of resistance.

Amongst the gram positives there was uniform susceptibility to Fosfomycin and Vancomycin. All the Staphylococcal isolates were also sensitive to Nitrofurantoin while $25 \%$ of Enterococcus sp. were resistant to the same. This implies that Fosfomycin and Nitrofurantoin are still showing promising results in terms of efficacy with added advantages that they are comparatively cheaper and are available in oral formulations for prescription in the outpatient setting. The current study demonstrates poor coverage by commonly prescribed Co-trimoxazole and Norfloxacin which is the same finding from previous studies ${ }^{1,2,15}$. Although Norfoxacin has been incorporated in the treatment options according to CLSI, we cannot continue with this antibiotic because of its poor coverage as per our results ${ }^{17}$.

Increasing antimicrobial resistance even in uncomplicated UTI as published in various 
studies is a matter of concern. From the above study we note that many of the commonly prescribed oral antibiotics are ineffective against even the common pathogens. More than $90 \%$ of pathogens in our study are already MDR. This puts pressure on the physicians to prescribe parenteral antibiotics, which can result in poor patient compliance because of cost factors and need for hospital admission. This further spirals the problem of emergence of antimicrobial resistance. Thus we have observed that there are limited options for prescribing oral antibiotics in the OPD setting.

Overall Fosfomycin is the most potent drug against both the Gram positive and Gram negative groups followed by Nitrofurantoin. In one study Fosfomycin had poor coverage in the gram positive group but our study showed that it is $100 \%$ effective against Enterococcus and Staphylococcus species $^{18}$.

There are few limitations in this study like small study population, lack of information on comorbidities and absence of radiological information to diagnose and correlate any anatomical findings.

\section{CONCLUSION}

Similar to the known fact, our study shows that $E$. coli is still the commonest bacteria responsible for lower urinary tract infection. Overall the prevalence of MDR is increasing however Fosfomycin or Nitrofurantoin can be the considered for starting empirical antibiotic therapy.

\section{ACKNOWLEDGMENTS}

We thank all the staff of Bacteriology laboratory, Department of Microbiology.

\section{CONFLICT OF INTEREST}

The authors declare that there is no conflict of interest.

\section{AUTHORS' CONTRIBUTION}

$M A, A S$ and FK designed the study. PK and $B C$ analyzed the data and PK wrote the manuscript. AK supervised and reviewed the manuscript. All the authors approved the manuscript.

\section{FUNDING}

None.

\section{ETHICS STATEMENT}

Informed and written consent was taken from each patient before specimen collection and processing and that the results may be included in the study.

\section{DATA AVAILABILITY}

All datasets generated or analyzed in this research are included in the manuscript.

\section{REFERENCES}

1. Haque R, Akter ML, Salam MA. Prevalence and susceptibility of uropathogens: a recent report from a teaching hospital in Bangladesh. BMC Res Notes. 2015;8:416.doi: 10.1186/s13104-015-1408-1

2. Sultan A, Rizvi M, Khan F, Sami H, Shukla I, Khan HM. Increasing antimicrobial resistance among uropathogens: Is fosfomycin the answer? Urol Ann. 2015;7(1):26-30. doi: 10.4103/0974-7796.148585

3. Rizvi M, Khan F, Shukla I, Malik A, Shaheen. Rising prevalence of antimicrobial resistance in urinary tract infections during pregnancy: Necessity for exploring newer treatment options. J Lab Physicians. 2011;3:98103. doi: 10.4103/0974-2727.86842

4. Gupta K. Addressing antibiotic resistance. Am J Med. 2002;113(Suppl 1A):29-34. doi: 10.1016/S00029343(02)01057-4

5. Mishra PP, Prakash V, Singh K, Mog H, Agarwal S. Bacteriological Profile of Isolates From Urine Samples in Patients of Benign Prostatic Hyperplasia and or Prostatitis Showing Lower Urinary Tract Symptoms. J Clin Diagn Res. 2016;10(10).

6. Parsons JK. Benign prostatic hyperplasia and male lower urinary tract symptoms: epidemiology and risk factors. Current Bladder Dysfunction Reports. 2010;5(4):212-18. doi: 10.1007/s11884-010-0067-2

7. Parsons JK, Bergstrom J, Silberstein J, BarrettConnor E. Prevalence and characteristics of lower urinary tract symptoms in men aged $>$ or $=80$ years. Urology. 2008;72(2):318-321. doi: 10.1016/j. urology.2008.03.057

8. Barkin J. Benign prostatic hyperplasia and lower urinary tract symptoms: evidence and approaches for best case management. Can J Urol. 2011;18:14-19.

9. Edlin RS, Heyns CF, Van Vuuren SP, Zarrabi AD. Prevalence of histological prostatitis in men with benign prostatic hyperplasia or adenocarcinoma of the prostate presenting without urinary retention. Int Braz J Urol. 2016;42(3):540-45.

10. Speakman MJ, Cheng X. Management of the complications of BPH/BOO. Indian J Urol. 2014;30(2):208-13. doi: 10.4103/0970-1591.127856

11. Forbes BA, Sahm DF, Weissfeld AS. Bailey \& Scott's Diagnostic Microbiology. $12^{\text {th }}$ ed ( $p$ 842-855). Missouri: 
Elsevier. 2007

12. Balaji V. Standard Operative Procedures Bacteriology. Antimicrobial Resistance Surveillance and Research Network. 2015.

13. Patricia TM. Bailey \& Scott's Diagnostic Microbiology. St. Louis, Missouri: Elsevier. 2014.

14. Winn WC, Allen SD, Allen S, et al. Woods Koneman's Textbook of Diagnostic Microbiology. 6th ed. Philadelphia: Lippincott Williams and Wilkins. 2006.

15. Baral P, Neupane S, Marasini PB, Ghimire KR, Lekhak B, Shrestha $B$. High prevalence of multidrug resistance in bacterial uro-pathogens from Kathmandu, Nepal. BMC Research Notes. 2012;5:38. doi: 10.1186/1756-05005-38
16. Comparative Prevalence of Antimicrobial Resistance in Community-Acquired Urinary Tract Infection Cases from Representative States of Northern and Southern India Journal of Clinical and Diagnostic Research. 2014;8(9):DC09-DC12.

17. Clinical and Laboratory Standards Institute. Performance standards for antimicrobial disk susceptibility tests. Wayne Pa; Clinical and Laboratory Standards Institute: $29^{\text {th }}$ edition M100. 2019.

18. Rubin RH, Beam TR Jr, Stamm WE. An approach to evaluating antibacterial agents in the treatment of urinary tract infection. Clin Infect Dis. 1992;14(Suppl 2): S246 51. doi: 10.1093/clinids/14.Supplement_2. S246 